\title{
Auswirkungen des Klimawandels auf die zukünftige Grundwassernutzung - Betroffenheiten, Handlungsbedarfe und Lösungsansätze
}

\author{
Steffen Bender ${ }^{1}$ Markus Groth ${ }^{1} \cdot$ Elisabeth Viktor ${ }^{1}$ \\ Eingegangen: 11. Mai 2020 / Überarbeitet: 16. September 2020 / Angenommen: 7. Dezember 2020 / Online publiziert: 21. Dezember 2020 \\ (c) Der/die Autor(en) 2020
}

\section{Zusammenfassung}

Die Ergebnisse regionaler Klimaprojektionen für Deutschland weisen auf eine Zunahme der mittleren Lufttemperatur und eine innerjährliche Verschiebung der Niederschläge - mit feuchteren Wintern und trockeneren Sommern - hin. Darüber hinaus werden sich regional die Häufigkeit, Intensität und Dauer von Hitzewellen, Trockenperioden und Starkregenereignissen weiter erhöhen. Durch diese Veränderungen wird sich auch der Jahresgang der Grundwasserneubildung ändern. Als Folge dessen können sich Änderungen bei den hohen, mittleren und tiefen Grundwasserständen, Grundwasserschwankungsbreiten und dem Grundwasserdargebot ergeben. Aber nicht nur die Ressource Grundwasser wird durch die Folgen des Klimawandels betroffen. Auch die gesamte Infrastruktur - von der Förderung bis zur Verteilungsleitung zum Kunden kann beeinträchtigt werden. Neben den direkten Einflüssen sind auch indirekte Beeinflussungen durch Kaskadeneffekte - beispielsweise ausgehend vom Energiesektor - möglich. Darum gilt es integrative, ganzheitliche und systemische Lösungen zu erarbeiten, um die Funktionalität der kritischen Infrastruktur dauerhaft auch unter Berücksichtigung der Folgen des Klimawandels gewährleisten zu können.

Schlüsselwörter Klimawandel $\cdot$ Wasserversorgung $\cdot$ Kritische Infrastruktur $\cdot$ Anpassung

\section{Climate change impacts on groundwater use-impacts and action needs}

\begin{abstract}
The results of regional climate projections for Germany show an increase in mean air temperature and an intra-annual shift in precipitation - with wetter winters and drier summers. Additionally, the frequency, intensity, and duration of heat waves, dry periods, and heavy rainfall events will increase regionally. These changes will also alter the annual distribution of groundwater recharge. As a result, high, mean, and low groundwater levels, the extent of groundwater level fluctuations, and available groundwater resources may change. However, it is not only the resource that is affected by climate change impacts. The entire infrastructure-from production to the service line to the customer-can also be affected by these impacts. In addition to direct influences, indirect influences are also possible through cascading effects—for example from the energy sector. Therefore, integrative, holistic, and systemic solutions have to be developed in order to guarantee the functionality of the critical infrastructure in the long term and with consideration of climate change impacts.
\end{abstract}

Keywords Climate change $\cdot$ Impacts $\cdot$ Water supply $\cdot$ Critical infrastructure $\cdot$ Adaptation

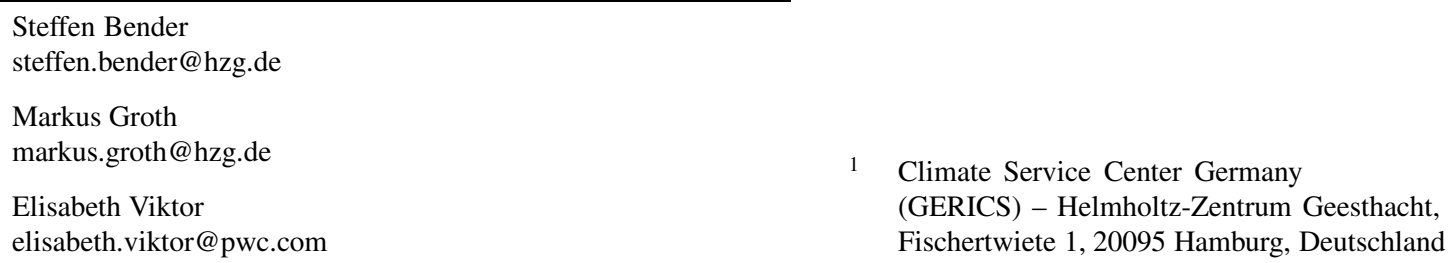




\section{Einleitung}

Trotz weltweiter Maßnahmen zum Klimaschutz ist seit fast sechs Jahrzehnten eine nahezu durchgehende Zunahme globaler $\mathrm{CO}_{2}$-Konzentrationen in der Atmosphäre zu beobachten. Dieser Trend wird von ebenfalls steigenden globalen Durchschnittstemperaturen begleitet (IPCC 2014), die im Vergleich zur vorindustriellen Zeit um rund $1{ }^{\circ} \mathrm{C}$ gestiegen sind (IPCC 2018). In Deutschland hat sich die mittlere Lufttemperatur von 1881 bis 2018 sogar bereits um $1,5^{\circ} \mathrm{C}$ erhöht und die mittlere jährliche Niederschlagsmenge hat um rund 8,7\% zugenommen (Umweltbundesamt 2019). Bei den Niederschlagsmengen treten auf regionaler und lokaler Ebene auch größere Abweichungen sowohl in der $\mathrm{Zu}$ - als auch in der Abnahme auf. Die Beobachtungen zeigen eine generelle innerjährliche Verschiebung der Niederschläge hin zu feuchteren Wintern und geringfügig trockeneren Sommern (DWD 2017). Zusätzlich sind Veränderungen bei den Niederschlagsextremen zu erkennen. Diese sind räumlich jedoch sehr heterogen, sodass sich je nach Region und Jahreszeit unterschiedliche Trends zeigen (Kunz et al. 2016), die es immer individuell zu untersuchen gilt. Van den Besselaar et al. (2012) beschreiben für den Zeitraum von 1951 bis 2010 für Europa eine Verringerung der Wiederkehrperioden von fünf- bis 20-jährigen Starkregenereignissen um $21 \%$. Auf regionaler Ebene und bei Betrachtung anderer Zeitperioden zeigen sich davon aber auch Abweichungen. Zolina et al. (2008) geben für den Zeitraum von 1950-2004 für weite Teile Westdeutschlands eine signifikante Abnahme der Starkniederschläge im Sommerhalbjahr um bis zu $8 \%$ pro Jahrzehnt an. Malitz et al. (2011) berechnen bei ihrer Betrachtung für Deutschland (Vergleich der Perioden 1951-2000 mit 1901-1950) dagegen eine mittlere Zunahme von $13 \%$. Für das Winterhalbjahr geben Malitz et al. (2011) und Zolina et al. (2008) eine mittlere Zunahme der Starkniederschläge in Deutschland von +5 bis $+22 \%$ an. Auf regionaler Ebene sind geringe Abnahmen für einige Regionen in der Mitte und Norddeutschland dokumentiert (Zolina et al. 2008; Trömel und Schönwiese 2007). Die Zunahme von Dürreperioden in weiten Teilen Deutschlands verdeutlicht zusätzlich die Veränderungen in der zeitlichen Niederschlagsverteilung (Umweltbundesamt 2019; IPCC 2018). Auch für Niederschläge in Form von Schnee und Hagel wurden bereits Veränderungen beobachtet. Mohr und Kunz (2013) sowie Kunz et al. (2016) zeigen beispielsweise eine leichte Zunahme des Hagelpotenzials und eine Abnahme der Schneedeckendauer.

Da der Klimawandel alle Kompartimente des hydrologischen Wasserkreislaufs betrifft, werden sich auf längere Sicht Veränderungen in den Grundwasserreservoiren ergeben. Für die Grundwasserbewirtschaftung liegt dabei das Hauptaugenmerk auf dem sich ändernden zeitlichen Verlauf der Grundwasserneubildung sowie den Veränderungen bei Schadstoffeinträgen einschließlich der Salzwasserintrusionen im Küstenraum (Feseker 2007; Ferguson und Gleeson 2012). Vor diesem Hintergrund besteht von Seiten der Wasserwirtschaft ein besonderes Interesse an Informationen über die zukünftige Häufigkeit, Intensität und Dauer von extremen Witterungsperioden wie Dürren, Hitzewellen und Starkregenereignissen.

Auf die wachsende Bedeutung der Folgen des Klimawandels und die Notwendigkeit einer vorausschauenden Umsetzung von ausreichend dimensionierten Anpassungsmaßnahmen weist auch das Weltwirtschaftsforum hin. Basierend auf den Einschätzungen von über 750 Experten und Entscheidungsträgern weltweit, zeigt der jüngste Bericht über die globalen Risiken (World Economic Forum 2020), dass im 10-Jahres-Ausblick der Umfrage erstmals die fünf größten globalen Risiken, gemessen an der Wahrscheinlichkeit, alle Umweltrisiken sind. Genannt sind dort insbesondere i) extreme Wetterereignisse mit erheblichen Schäden an Eigentum, Infrastruktur und Verlust von Menschenleben, ii) das Scheitern der Klimaschutz- und Anpassungsmaßnahmen von Regierungen und Unternehmen, iii) vom Menschen verursachte Umweltschäden und Katastrophen, iv) ein schwerwiegender Verlust an Artenvielfalt und der Zusammenbruch von Ökosystemen (Land und Wasser) sowie v) große Naturkatastrophen.

\section{Die Bedeutung regionaler Klimainformationen}

Um die Folgen des Klimawandels für eine Region genau betrachten zu können, werden regionale Klimaprojektionen benötigt (vgl. dazu Brasseur et al. 2016; Wagner et al. 2013; Jacob et al. 2012). Diese zeigen in sich sowohl schlüssige als auch mögliche, zukünftige Entwicklungen unter gegebenen Randbedingungen des Klimasystems auf. Die Arbeit mit Praxispartnern zeigt darüber hinaus, dass die bereitgestellten Informationen nur dann eine ausreichende Akzeptanz erfahren, wenn sie bedarfsorientiert und mit Interpretationshilfen versehen sind (Tart et al. 2020; Bender et al. 2019).

Für den 5. Assessment Report des IPCC (2014) wurde ein neues Konzept zu Erstellung globaler Emissionsszenarien entwickelt (Meinshausen et al. 2011). Dieses beruht abweichend von der vorherigen Praxis auf der Nutzung sogenannter „repräsentativer Konzentrationspfade“ (Moss et al. 2010). Hierbei wird der Strahlungsantrieb der Klimamodelle - das Maß für die Veränderung der Energiebilanz der Erde durch externe Faktoren - zum Ende des 21. Jahrhunderts definiert. Welche sozioökonomischen Entwicklungen für diesen Verlauf verantwortlich sind, spielt dabei nur eine untergeordnete Rolle. Diese Szenarien werden nach der Änderung des Strahlungsantriebs (der Netto-Strahlungsflussdich- 

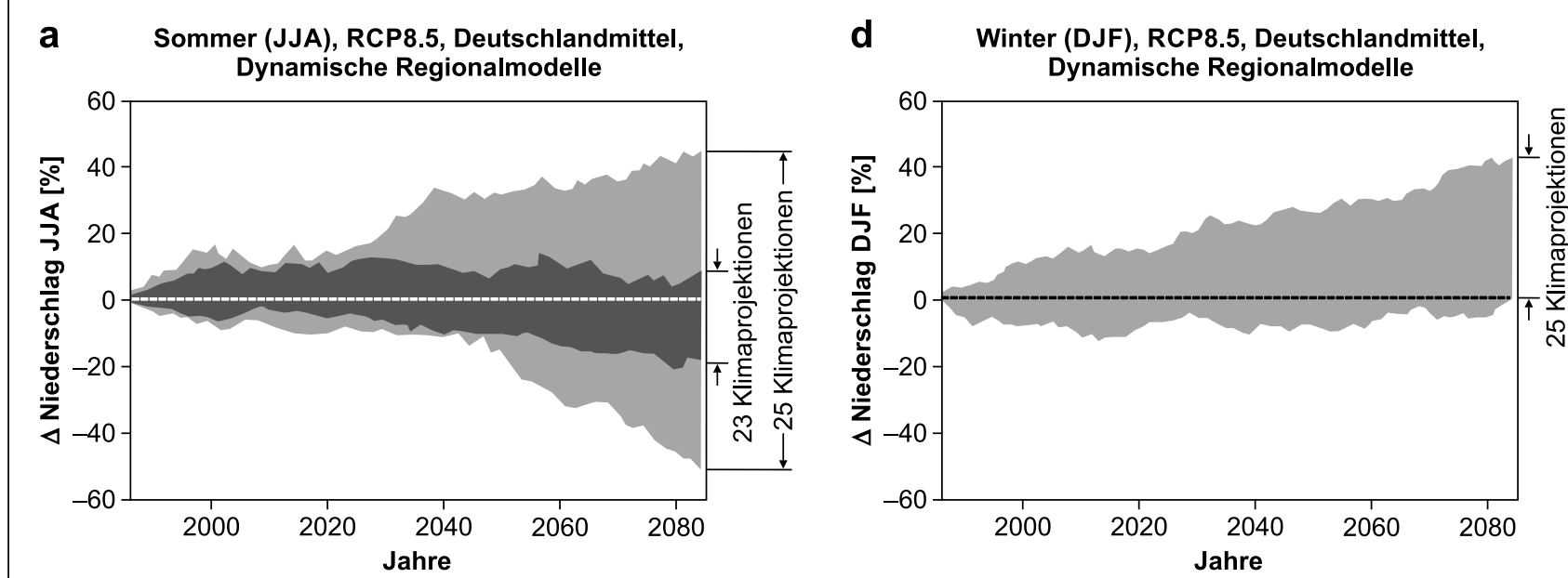

b Sommer (JJA), RCP8.5, Deutschlandmittel,

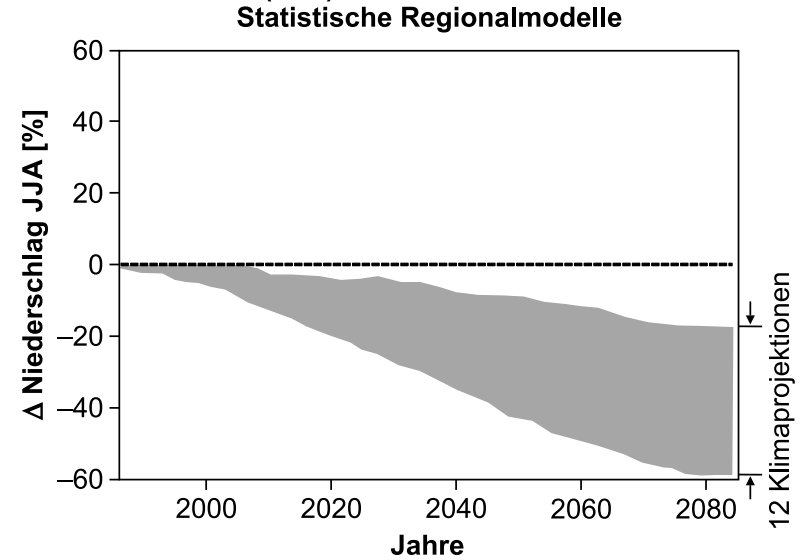

e Winter (DJF), RCP8.5, Deutschlandmittel,

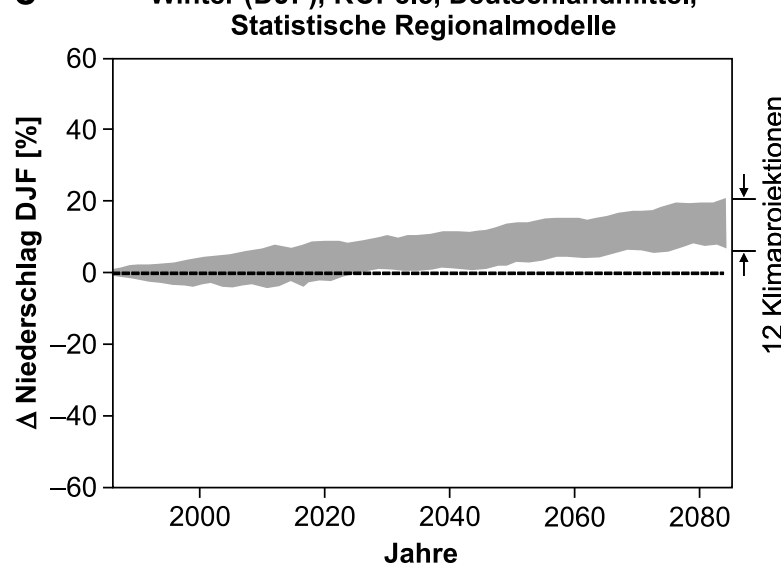

C Sommer (JJA), RCP8.5, Deutschlandmittel, Alle Regionalmodelle
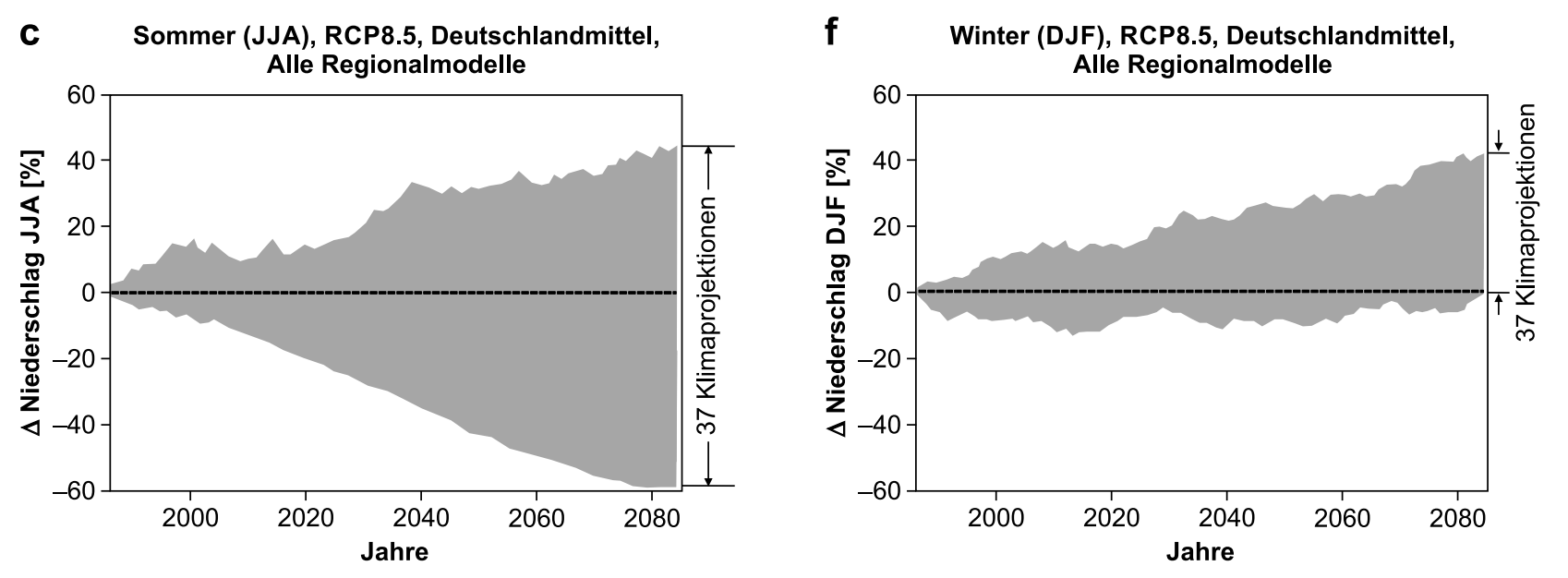

Abb. 1 Projizierte Änderungen der Niederschlagsmengen im Deutschlandmittel für das RCP8.5 (30-jähriges gleitendes Mittel: 1986-2085 minus 1971-2000). (Nach ReKliEs-De 2017, verändert). Änderungen der Niederschlagsmengen im Sommerhalbjahr (Juni, Juli, August) als Ergebnisbandbreite von a dynamischen Regionalmodellen, b statistischen Regionalmodellen sowie $\mathbf{c}$ dynamischen und statistischen Regionalmodellen. Änderungen der Niederschlagsmengen im Winterhalbjahr (Dezember, Januar, Februar) als Ergebnisbandbreite von d dynamischen Regionalmodellen, e statistischen Regionalmodellen sowie $\mathbf{f}$ dynamischen und statistischen Regionalmodellen (37 Klimaprojektionen)

Fig. 1 Projected changes of precipitation amount for Germany in average for RCP8.5 (30-year moving average: 1986-2085 minus 1971-2000). (After ReKliEs-De 2017, modified). Changes in precipitation amounts in summer half-year (June, July, August) as a result range of a dynamic regional models, $\mathbf{b}$ statistical regional models, and $\mathbf{c}$ dynamic and statistical regional models. Changes in precipitation quantities in winter half-year (December, January, February) as a result of $\mathbf{d}$ dynamic regional models, $\mathbf{e}$ statistical regional models, and $\mathbf{f}$ dynamic and statistical regional models 


\begin{tabular}{|c|l|c|c|r|}
\hline Änderung & $\begin{array}{l}\text { Klimakennwerte } \\
\text { (Ensemble-Median) }\end{array}$ & RCP2.6 & RCP8.5 & $\begin{array}{l}\text { Vorteil RCP2.6 } \\
\text { gegenüber RCP8.5 }\end{array}$ \\
\hline & Jährliche mittlere Temperatur $\left[{ }^{\circ} \mathrm{C}\right]$ & +1 & +4 & 4 mal kleiner \\
\hline & Anzahl der Sommertage $\left(\mathrm{T}_{\max }>25^{\circ} \mathrm{C}\right)$ & +10 & +46 & 4,5 mal kleiner \\
\hline & Anzahl der Hitzetage $\left(\mathrm{T}_{\max }>30^{\circ} \mathrm{C}\right)$ & +4 & +19 & 4 bis 5 mal kleiner \\
\hline & Anzahl der Eistage $\left(\mathrm{T}_{\max }<0^{\circ} \mathrm{C}\right)$ & -8 & -19 & 2,5 bis 5 mal kleiner \\
\hline
\end{tabular}

\begin{tabular}{|c|l|c|c|c|}
\hline Änderung & Klimakennwerte & RCP2.6 & RCP8.5 & $\begin{array}{l}\text { Vorteil RCP2.6 } \\
\text { gegenüber RCP8.5 }\end{array}$ \\
\hline & Anzahl nasse Tage, Sommer $(\mathrm{N}>20 \mathrm{~mm})$ & $-30 \%$ bis $+30 \%$ & $-50 \%$ bis $+120 \%$ & 3 bis 4 mal kleiner \\
\hline & Niederschlagsmenge, Sommer & $-30 \%$ bis $+10 \%$ & $-60 \%$ bis $+10 \%$ & 2 bis 3 mal kleiner \\
\hline & Anzahl nasse Tage, Winter $(\mathrm{N}>20 \mathrm{~mm})$ & $0 \%$ bis $+40 \%$ & $+20 \%$ bis $+130 \%$ & 4 bis 5 mal kleiner \\
\hline & Niederschlagsmenge, Winter & $-15 \%$ bis $+18 \%$ & $0 \%$ bis $+40 \%$ & 2 bis 3 mal kleiner \\
\hline
\end{tabular}

Abb. 2 Vergleich (2071-2100 mit 1971-2000) der Veränderungen ausgesuchter temperatur- und niederschlagsbasierter Kennwerte für Deutschland. (Datenquelle: ReKliEs-De 2017)

Fig. 2 Comparison (2071-2100 with 1971-2000) of changes in selected temperature- and precipitation-based parameters for Germany. (Data source: ReKliEs-De 2017)

te an der Tropopause), bis in das Jahr 2100 gegenüber dem vorindustriellen Antrieb benannt. So führt beispielsweise der Konzentrationspfad des RCP8.5 zu einem Strahlungsantrieb von $8,5 \mathrm{~W} / \mathrm{m}^{2}$ im Jahre $2100 \mathrm{im}$ Vergleich zum Jahr 1850. Da hier ein kontinuierlicher Anstieg der Treibhausgasemissionen angenommen wird, hat sich dafür der Begriff „Weiter-wie-bisher“-Szenario etabliert (Brasseur et al. 2016). Eine moderatere Entwicklung mit einigen erfolgreichen Klimaschutzmaßnahmen wird durch das RCP4.5 wiedergegeben. Der Konzentrationspfad des RCP2.6 basiert dagegen auf der Umsetzung sehr ambitionierter Maßnahmen zur Reduktion von Treibhausgasemissionen und spiegelt dies wider, was in der Regel als „Klimaschutz“-Szenario bezeichnet wird.

Um die Folgen des Klimawandels in wasserwirtschaftliche Planungsprozesse und Managementstrategien integrieren zu können, steht Deutschland ein weltweit einzigartiger Datensatz regionaler Klimainformationen zur Verfügung. Dieser basiert zum einen auf den Daten der internationalen Initiative EURO-CORDEX (Coordinated Downscaling Experiment - European Domain), wo koordinierte Simulationen mit einer horizontalen Auflösung von $12 \mathrm{~km}$ für Europa basierend auf unterschiedlichen RCPs durchgeführt werden (Jacob et al. 2014). Zum anderen fließen Ergebnisse des BMBF-Forschungsprojekts ReKliEs-De (Regionale Klimaprojektionen Ensemble für Deutschland) mit ein, die weitere Informationen für die Szenarien RCP2.6 und RCP8.5 beisteuern (ReKliEs-De 2017). Generell muss bei der Nutzung von Klimainformationen stets darauf geach- tet werden, ob die Aussagen über die Klimaänderungen signifikant und robust sind. Dazu wurde von Pfeifer et al. (2015) ein entsprechendes Vorgehen entwickelt. Aber auch die sachgerechte Interpretation und Anwendung der Ergebnisse ist vor allem für Anpassungsmaßnahmen an die Folgen des Klimawandels von großer Bedeutung (Bender und Jacob 2016).

Alle regionalen Klimaprojektionen zeigen für Deutschland bis zum Ende des 21. Jahrhunderts eine Fortsetzung des bereits beobachteten Temperaturanstiegs. In Bezug auf Hitzewellen ist es sehr wahrscheinlich, dass diese aufgrund der Zunahme der mittleren Temperatur zukünftig mit einer größeren Häufigkeit und längeren Dauer auftreten werden (Deutschländer und Mächel 2016; Collins et al. 2013).

Im Vergleich zur Referenzperiode 1971-2000 zeigen die Ergebnisse für den Zeitraum 2071-2100 eine Erhöhung der bodennahen Lufttemperatur zwischen 3,1 und $4,9^{\circ} \mathrm{C}$ (RCP8.5; Weiter-wie-bisher-Szenario) beziehungsweise zwischen 0,8 und $1,8^{\circ} \mathrm{C}$ (RCP2.6; Klimaschutz-Szenario). Bei der Änderung der mittleren Jahresniederschlagsmengen zeigen sich dagegen uneinheitliche Signale. Die simulierten Änderungen im RCP8.5 betragen teilweise mehr als $\pm 20 \%$, im RCP2.6 $\pm 10 \%$ (ReKliEs-De 2017).

Regionale Klimaprojektionen der EURO-CORDEX-Initiative zeigen für Deutschland eine robuste und signifikante Zunahme der Häufigkeit von Starkregenereignissen im Herbst und Winter von bis zu $25 \%$. Für das Szenario RCP4.5 ergeben sich keine signifikanten Veränderungen (Jacob et al. 2014). Die ReKliEs-De-Ergebnisse verdeutli- 
chen zudem für Deutschland, dass die Niederschlagsmengen von Starkregen im RCP8.5 über fünfmal höher liegen als im RCP2.6 (ReKliEs-De 2017). Betrachtet man die Ergebnisse der Veränderungen saisonaler Niederschlagsmengen etwas differenzierter, so zeigt sich insbesondere bei den Veränderungen der Niederschlagsmengen im Sommer deutlich der Einfluss der verwendeten, regionalen Klimamodelle. Hierbei sind die Ergebnisse statistischer Regionalmodelle deutlich trockener als die dynamischer Regionalmodelle. Aber auch bei den dynamischen Regionalmodellen gibt es Ausreißer, die extremere Ergebnisse liefern (Abb. 1). Im Winter liegen die Ergebnisse dynamischer Regionalmodelle sowohl über als auch vereinzelt unter denen statistischer Regionalmodelle. Folglich sollten alle Fragestellungen, die zukünftige Grundwasserneubildungswerte aus Klimainformationen ableiten, Ergebnisse beider Regionalmodellarten verwenden, um das Dargebot oder die Wasserstände nicht zu unter- oder überschätzen. Damit können beispielsweise abgestufte Vorgehensweisen auf Basis von Bemessungsgrößen entwickelt werden, die ein zeitnahes Eingreifen bei sich ändernden Rahmenbedingungen, wie Wasserständen, ermöglichen.

Wie positiv sich Klimaschutzbemühungen auf zukünftige klimatische Veränderungen auswirken können, zeigt der direkte Vergleich von temperatur- und niederschlagsbasierten Kennwerten - wie der Anzahl der Sommertage $\left(\mathrm{T}_{\max }\right.$. $\left.25^{\circ} \mathrm{C}\right)$ und Hitzetage $\left(\mathrm{T}_{\max } 30^{\circ} \mathrm{C}\right)$ oder die Niederschlagsmengen im Sommer oder Winter - zwischen den Ergebnissen des „Klimaschutz-Szenarios“ (RCP2.6) und denen des „Weiter-wie-bisher-Szenarios“ (RCP8.5) für die Perioden 2071-2100 und 1971-2000. Durch eine deutliche Verminderung der Treibhausgasemissionen kann das Auftreten von sowohl temperatur- als auch niederschlagsbasierten Ereignissen um den Faktor zwei bis fünf verringert werden (Abb. 2). Dadurch könnten sowohl potenzielle Schäden an der Infrastruktur reduziert als auch wasserbedarfsorientierte Herausforderungen abgeschwächt werden.

\section{Neue Herausforderungen für die Wasserwirtschaft}

Der Klimawandel und seine Folgen können alle Komponenten des Wasserkreislaufs direkt oder indirekt beeinflussen. Darüber hinaus sorgen der demographische Wandel, Veränderungen in den Stadtstrukturen und Wohnformen, sich ändernde Haushaltsgrößen oder neues Freizeitverhalten für eine Veränderung der Verbrauchsspitzen. Somit können sich in Verbindung mit den Folgen des Klimawandels aus Sicht des Wasserversorgers temporär neue Herausforderungen ergeben, was die Bereitstellung ausreichender Wassermengen aber auch die Aufrechterhaltung der geforderten Wasserqualität betrifft (Riedel 2019; Whitehead et al. 2009). Dies trifft insbesondere Wasserversorger, die ausschließlich Wasser aus Quellen oder oberflächennahen Grundwasserleitern beziehen. Schon heute ist zu erkennen, dass Wetterereignisse mit extremer Ausprägung die Ziele und Aufgaben der öffentlichen Trinkwasserversorgungen direkt oder indirekt beeinträchtigen können. Dies kann im ungünstigsten Fall sogar zu einem zeitweisen Ausfall von Gewinnungsanlagen und Versorgungseinrichtungen führen (DVGW 2018). Abhängig von der orts- und infrastrukturabhängigen Vulnerabilität müssen die potenziell zu erwartenden StörfallSzenarien in der Zukunft analysiert und geeignete präventive Anpassungsmaßnahmen entwickelt werden. Tab. 1 gibt einen Überblick möglicher witterungsbedingter Störszenarien. Bei der Planung und Umsetzung von Maßnahmen gilt es zur berücksichtigen, dass die natürliche zeitliche Entwicklung der Grundwasserstände zusätzlich von anthropogenen Eingriffen weiter beeinflusst werden kann. Somit ist in jedem Fall eine Einzelfallprüfung notwendig.

Eine für grundwassernutzende Wasserversorger wichtige Größe - neben der Art, Mächtigkeit und Tiefe des Grundwasserleiters sowie bodenhydraulischen Kennwerten der Deckschichten - ist der jahreszeitliche Gang der Grundwasserneubildung. Deren Verlauf wird neben anthropogenen Grundwasserentnahmen und -einleitungen durch weitere Faktoren beeinflusst. Hierbei unterscheidet man zwischen i) den direkten klimatischen Einflussfaktoren (wie Temperatur, Sonnenscheindauer, Wind und Niederschlägen), ii) den indirekten klimatischen Faktoren (wie Evaporation, Transpiration und vegetationsabhängigen Größen - Länge der Vegetationsperiode oder Blattflächenindex) und iii) den bodenabhängigen Faktoren (wie Versiegelung, Infiltrationsvermögen, Wassergehalt, Austrocknungs- und Wiederbefeuchtungszyklen, Grundwasserflurabstand). Vor allem oberflächennahes Grundwasser aus räumlich eng begrenzten Grundwasserleitern und mit kleinem Speichervolumen reagiert hochsensibel auf längere Trockenperioden und Hitzewellen, wobei die Schwankungsbreiten der Grundwasserstände stark vom Grundwasserleitertyp geprägt werden. Grundwasser reagiert auch auf Niederschlagsschwankungen mit einer mehr oder weniger großen Zeitverzögerung. Nachhaltige Veränderungen beruhen somit nicht auf den Witterungsverläufen eines Jahres, vielmehr spielen die Grundwasserstände der Vorjahre beziehungsweise die Abfolge von Nass- und Trockenjahren eine wichtige Rolle (Hergesell 2017). Allerdings zeigen Beobachtungen auch, dass nicht nur die vorangegangenen Jahre eine Rolle spielen, da die tiefsten Grundwasserstände nicht immer direkt im Folgejahr eines Trockenjahres auftreten (Abb. 3). Die Schwankungsbreiten der Grundwasserstände in den Folgejahren nach Trocken- und Nassjahren zeigen auch keine eindeutige Clusterbildung, was darauf hindeutet, dass die Wechselwirkungen zwischen Niederschlagsmengen und Grundwasserständen deutlich komplexer sind. In Verbin- 
Tab. 1 Störfallszenarien für Versorgungsanlagen, die ausschließlich Grundwasser nutzen

Table 1 Incident scenarios for water supply facilities that exclusively use groundwater

\begin{tabular}{|c|c|c|}
\hline Extremereignis & Auswirkungen auf die Wasserversorgung & Potenzielle Maßnahmen \\
\hline $\begin{array}{l}\text { Trockenperioden } \\
\text { (Grundwasser) }\end{array}$ & $\begin{array}{l}\text { Über mehrere Jahre fallender Grundwasserspiegel } \\
\text { Brunnen können nicht mehr wie gewünscht betrieben } \\
\text { werden } \\
\text { Erhöhung der Spitzenverbräuche }\end{array}$ & $\begin{array}{l}\text { Monitoring (präventiv) } \\
\text { Stützung von Naturschutzgebieten } \\
\text { Reduktion und Verlagerung der Entnahme }\end{array}$ \\
\hline $\begin{array}{l}\text { Trockenperioden } \\
\text { (Quellen) }\end{array}$ & Rückgang der Quellschüttung & $\begin{array}{l}\text { Monitoring (präventiv) } \\
\text { Reduktion mit Verlagerung der Entnahme }\end{array}$ \\
\hline Hitzewellen & $\begin{array}{l}\text { Steigende Rohwasser- und Trinkwassertemperatur } \\
\text { Erhöhung der Spitzenverbräuche } \\
\text { Brandgefahr auf dem Wasserwerksgelände }\end{array}$ & $\begin{array}{l}\text { Neue Konzepte für die Verlegung von Wasserleitungen } \\
\text { Stagnation von Trinkwasser im Netz minimieren } \\
\text { Beschränkung der Wasserverwendung im Außenbereich } \\
\text { Ausarbeitung von Maßnahmenplänen in Zusammenarbeit } \\
\text { mit der Feuerwehr }\end{array}$ \\
\hline $\begin{array}{l}\text { Starkregen } \\
\text { (Grundwasser) }\end{array}$ & $\begin{array}{l}\text { Eingeschränkte Zuwegung } \\
\text { Überschwemmung und Verschmutzung der Infrastruktur } \\
\text { oder elektrischer Anlagen } \\
\text { Flutung des Brunnenschachtes } \\
\text { Trübungseinbrüche infolge von Undichtigkeiten oder } \\
\text { Kurzschlussströmungen am Fassungsbauwerk }\end{array}$ & $\begin{array}{l}\text { Detaillierte Bewertung der einzelnen Standorte } \\
\text { Aufrechterhaltung/Schaffung redundanter Systeme }\end{array}$ \\
\hline $\begin{array}{l}\text { Starkregen } \\
\text { (Quellen) }\end{array}$ & $\begin{array}{l}\text { Eingeschränkte Zuwegung } \\
\text { Überschwemmung und Verschmutzung der Infrastruktur } \\
\text { oder elektrischer Anlagen } \\
\text { Trübungseinbrüche infolge von Undichtigkeiten oder } \\
\text { Kurzschlussströmungen am Fassungsbauwerk möglich }\end{array}$ & $\begin{array}{l}\text { Detaillierte Bewertung der einzelnen Standorte } \\
\text { Aufrechterhaltung/Schaffung redundanter Systeme }\end{array}$ \\
\hline Sturmereignisse & $\begin{array}{l}\text { Blockade der Zuwege } \\
\text { Beschädigung der Infrastruktur oder elektrischer Anlagen } \\
\text { Eintrag von Oberflächenwasser über Undichtigkeiten an } \\
\text { und in Bauwerken (Wasserbehälter, Stollen, etc.) }\end{array}$ & $\begin{array}{l}\text { Detaillierte Bewertung der einzelnen Standorte } \\
\text { Aufrechterhaltung/Schaffung redundanter Systeme }\end{array}$ \\
\hline
\end{tabular}

dung mit den vielen möglichen natürlichen Veränderungen einzelner oder mehrerer Komponenten des Wasserkreislaufs, sind deshalb genaue Abschätzungen zukünftiger, möglicher Veränderungen von Grundwasserganglinien aktuell nur schwer möglich, beziehungsweise mit großen Ergebnisbandbreiten verbunden. Betrachtet man dazu die Ergebnisse regionaler Klimaprojektionen der EUROCORDEX-Initiative für Deutschland, so zeigen diese einen robusten und signifikanten Anstieg der Temperatur und eine nahezu unveränderte jährliche Niederschlagsumme (Jacob et al. 2014). Allerdings wird sich die bereits heute schon beobachtete innerjährliche Umverteilung von geringeren Niederschlagsmengen im Sommer zu größeren im Frühling, Herbst und Winter (DWD 2017) weiter fortsetzen. Durch den Anstieg der Temperatur nimmt jedoch die vegetationsfreie Zeit ab (Zebisch et al. 2005). So kann der mittlere Start der Vegetationsperiode regionsspezifisch um mehrere Wochen früher erfolgen und sich dadurch die mittlere Vegetationslänge um bis zu einen Monat erhöhen (Bender et al. 2018). Durch den damit gesteigerten Wasserbedarf der Pflanzen in Verbindung mit höheren Verdunstungsraten wird die Grundwasserneubildung in vielen Regionen abnehmen (Kunstmann et al. 2016). Hattermann et al. (2008) ermittelten dazu für das Einzugsgebiet der Elbe einen Rückgang der Neubildungsmenge um rund $30 \%$.
Durch den Anstieg der Temperatur im Jahresmittel werden die Winterniederschläge zukünftig vermehrt als Regen fallen. Nach Kunstmann et al. (2016) werden besonders niedrige Höhenlagen bis $1000 \mathrm{~m}$ sowie bis $1500 \mathrm{~m}$ in alpinen Gebieten von einer starken Abnahme der Schneedeckendauer betroffen sein. Dadurch verringert sich die natürliche Retention des Niederschlags als Schnee, wodurch im Winter die Abfluss- und Infiltrationsmengen steigen und im Sommer weiter abnehmen werden.

Bedingt durch die beschriebenen Veränderungen einzelner oder mehrerer Komponenten des Wasserkreislaufs wird sich zukünftig vielerorts der Wasserstress erhöhen. Dies könnte insbesondere in schon heute trockenen Gebieten, wie Ost-Deutschland, Franken und Oberpfalz die Wasserversorgung vor noch größere Herausforderungen stellen. Neben den zeitlichen Wasserengpässen könnte sich langfristig auch die jährlich gespeicherte Wassermenge dauerhaft verringern (Bender et al. 2017; Green et al. 2011; Döll 2009). Dies wird sowohl Grundwasserstände und Quellschüttungen beeinflussen. Studien weisen darauf hin, dass in einigen Regionen die natürlichen Grundwasserspiegelschwankungen zunehmen werden (Brahmer 2006; Umweltbundesamt 2019). Genaue Abschätzungen über die zukünftige Entwicklung lokaler und regionaler Grundwasserstände sind, aufgrund der großen Ergebnis-Bandbreiten bei der Modellierung regionaler Klimaparamater und den Abwei- 


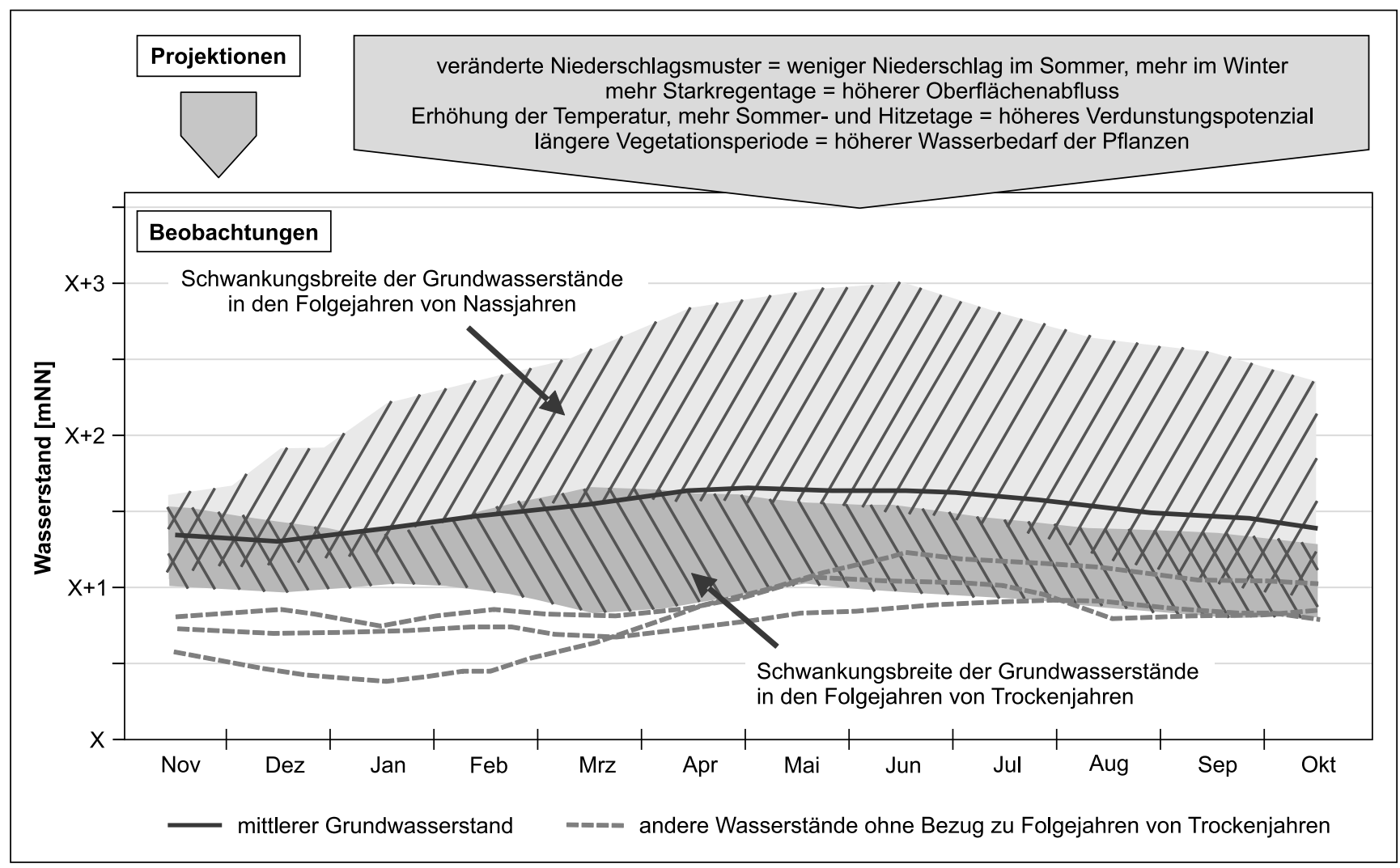

Abb. 3 Schematische Darstellung beobachteter Grundwasserschwankungen (1962-2014) an einer Referenzmessstelle (anthropogen-unbeeinflusst) im obersten Porengrundwasserleiter am Niederrhein (Nordrhein-Westfalen) sowie projizierter Veränderungen im lokalen Wasserkreislauf. Die Beobachtungen zeigen die Schwankungsbreiten der Grundwasserstände in den Folgejahren nach den jeweils fünf extremsten Nass- und Trockenjahren sowie die Ganglinien von Tiefstwasserständen ohne Bezug zu einem direkt zurückliegenden Trockenjahr

Fig. 3 Scheme of monitored groundwater levels (1962-2014) at a reference observation well in the uppermost porous aquifer in the Lower Rhine (North Rhine-Westphalia) as well as projected changes in the local water cycle. The observations show the fluctuation ranges of the groundwater levels in the following years after the five most extreme wet and dry years as well as groundwater hydrographs of low water levels without reference to a directly past dry year

chungen bei der Modellierung saisonaler Niederschlagsmengen, nur schwer möglich (Kunstmann et al. 2016).

Auch beim zeitlichen Verlauf von Quellschüttungen werden zukünftig Veränderungen erwartet, wobei die bisherigen Erkenntnisse nur daraufhin deuten, dass jede Quelle individuell betrachtet werden muss (HLUG 2014; Euler et al. 2009; BayLfW 2005).

Wasserversorger müssen ihren Blick aber nicht nur auf die Menge und Qualität von Rohwasser richten, auch die Bereitsstellung des Trinkwassers kann zukünftig zu einer Herausforderung werden. Obwohl die Rohrleitungen und ihr Inhalt durch die unteriridische Lage gegen direkte meteorologische Extremereignisse geschützt sind, können die steigenden Lufttemperaturen insbesondere bei Hitzewellen auch das Trinkwasser erwärmen. Forschungsergebnisse belegen, dass sich nicht nur das Rohwasser durch klimatische Einflüsse auf über $25^{\circ} \mathrm{C}$ erwärmen kann, sondern auch das Wasser auf dem Transportweg bis zum Verbraucher (Osmancevic et al. 2018; Merkel und Staben 2014). Durch die Erhöhung der Lufttemperatur, die Zunahme von Hitzewel- len und die weitere Versiegelung von Städten werden die Bodentemperaturen weiter ansteigen, was zusammen mit der Wärmelast in modernen Gebäuden eine weitere Zunahme der Trinkwassertemperatur fördern wird.

\section{Die Wasserversorgung als Teil der kritischen Infrastruktur}

\section{Die klimaresiliente Infrastruktur}

Die kritische Infrastruktur, einschließlich der Wasserversorgung, hat eine zentrale Bedeutung für das Gemeinwesen (BBK 2020). Sie bildet ein komplexes System mit sektorübergreifenden Aufgaben, wobei insbesondere an den vielfältigen Schnittstellen die Zuständigkeiten nicht immer klar definiert sind. Zudem reichen in der Praxis vielerorts die Notfallpläne nicht aus, um die vielfältigen Auswirkungen des Klimawandels hinsichtlich der Funktionsfähigkeit von Infrastrukturelementen zu berücksichtigen. Insbeson- 
dere positive und negative Rückkopplungen sind von zunehmender Bedeutung, sodass sich Verwundbarkeiten verschärfen und sich innerhalb der vernetzten Infrastrukturelemente auch sektorübergreifend weiterführen können (Forzieri et al. 2018; Groth et al. 2018; Laugé et al. 2015; Eusgeld et al. 2011). Darüber hinaus werden in vielen Teilbereichen immer mehr redundante Systeme eingespart und durch intelligente (,smarte“) Lösungen ersetzt, wodurch sich die Gefahr für Anfälligkeiten in Verbindung mit Systemausfällen erhöhen kann (Mikellidou et al. 2018; Groth et al. 2016).

Als Folge der schon heute spürbaren sowie der projizierten klimatischen Veränderungen ist eine zentrale systematische Herausforderung somit der Aufbau bzw. die Aufrechterhaltung einer widerstandsfähigen Infrastruktur. Dies steht in engem Zusammenhang mit dem beispielsweise auch in der Agenda 2030 formulierten Anspruch, Städte und Siedlungen widerstandsfähig und nachhaltig zu machen. Vor diesem Hintergrund sind nicht nur für Stadtverwaltungen und Wasserversorger, die sich aus den Folgen des Klimawandels ergebenen Herausforderungen von immer größerer Relevanz. Der IPCC-Sonderbericht „Global Warming of $1,5^{\circ} \mathrm{C}^{\prime \prime}$ (IPCC 2018) weist zudem darauf hin, dass bereits eine Erwärmung von $1,5^{\circ} \mathrm{C}$ im Vergleich zum vorindustriellen Niveau - definiert im Fünften Sachstandsbericht des IPCC (IPCC 2014) als Bezugszeitraum von 1850 bis 1900 - deutliche Risiken für viele urbane Handlungsfelder, darunter auch die Wasserversorgung, mit sich bringen kann. Darüber hinaus wird in dem Bericht gezeigt, dass eine Begrenzung der globalen Erwärmung auf $1,5^{\circ} \mathrm{C}$ möglich ist. Dazu ist allerdings ein konsequentes und schnelles Handeln notwendig, um die Netto-Treibhausgasemissionen in einem Zeitraum von 2040 bis 2050 weltweit auf null sinken zu lassen. Um diese Minderungen herbeizuführen sind jedoch grundlegende Transformationen in gesellschaftlich und wirtschaftlich bedeutenden Bereichen verbunden (Groth 2019). Dies umfasst neben ambitioniertem Klimaschutz auch die Anpassungen zentraler Versorgungsinfrastrukturen an die heute bereits spürbaren beziehungsweise nicht mehr zu vermeidenden zukünftigen Folgen des Klimawandels. Im Idealfall können dadurch auch positive Rückkopplungen erneuerbarer bzw. alternativer Energiequellen auf die Versorgungssicherheit (Redundanz-Kriterium) realisiert werden (Olfert et al. 2020). Für die zielgerichtete Umsetzung von Maßnahmen wird ein integratives Systemverständnis benötigt, in dem die komplexen Wechselwirkungen zwischen klimatischen und nicht-klimatischen Treibern, einschließlich der Wechselwirkungen und Rückkopplungen einzelner und mehrerer Infrastrukturelemente berücksichtigt werden (Mikellidou et al. 2018; Laugé et al. 2015). Einen Überblick über die Komplexität beispielsweise über den Wasser-Energie-Land-Nexus in Verbindung mit
Erkenntnissen basierend auf regionalen Klimainformationen geben Cremades et al. (2019).

\section{Die Verbindung von Strom- und Wasserversorgung}

Die klimatischen Veränderungen, die die Strom- und Wasserversorgung heute und zukünftig vor größere Herausforderungen stellen werden, sind der Anstieg der Durchschnittstemperatur, Hitzewellen, Dürreperioden und Extremniederschläge (Groth und Rose 2017). Der Wassersektor wird durch die genannten Veränderungen vor Herausforderungen gestellt und zwar direkt und indirekt. Die direkten Einflüsse führen zu Veränderungen der Verfügbarkeit, Quantität und Qualität der Ressource Wasser. Die indirekten Einflüsse beeinflussen alle Abläufe, die eine permanente Stromversorgung benötigen, wie die Wasserförderung und -aufbereitung, den Transport zu Zwischenspeichern oder dem Verbraucher sowie die Telekommunikation und EDV-Vernetzung von Steueranlagen. Groth et al. (2018) diskutieren dazu detailliert die Folgen für den Energiesektor in Deutschland. Betrachtet man dazu die Stabilität des Stromnetzes, so zeigen die Zahlen der Bundesnetzagentur, dass es einen direkten Zusammenhang zwischen dem Auftreten von extremen Wetterereignissen (Stürme, Starkregen, Hochwasser) und Unterbrechungen im Verteilernetz gibt (Stankoweit et al. 2017).

Experten aus dem Wasser- und Energiesektor weisen darauf hin, dass sowohl natürliche Faktoren als auch Entscheidungsstrukturen eine wichtige Rolle bei der Gewährleistung der Versorgungssicherheit spielen (Birkmann et al. 2010). Als eine der immer wichtiger werdenden Herausforderungen ist hierbei die stetig wachsende Vernetzung kritischer Infrastrukturelemente zu nennen, wobei immer mehr ,smarte Lösungen“ eingesetzt werden (Graumann 2017). Diese gilt es jedoch auf den Prüfstand zu stellen, um im Falle möglicher Teilsystemausfällen, insbesondere der Stromversorgung, nicht die Funktionalität von einem oder mehrerer wichtiger Teilkomponenten zu verlieren. Durch die fortschreitende Digitalisierung und Vernetzung entstehen zunehmend komplexere, multisektorale Versorgungssysteme (Mikellidou et al. 2018). Diese setzen sich aus einer Vielzahl unterschiedlicher Elemente zusammen, wobei jedes einzelne unterschiedlich stark von den verschiedenen Extremwetterereignissen beeinflusst wird. Generell wird es nie möglich sein, allen Arten von Störungen vorzubeugen. Die wichtigste Aufgabe der Versorgungsinfrastrukturen ist insbesondere die zuverlässige Bereitstellung unentbehrlicher Dienstleistungen, durch die sie in besonderer Weise mit der Gesellschaft verwoben sind. Daher kommt auch der Vermeidung des Überspringens von Ausfällen - die benachbarte Systeme oder auch andere Teile der kritischen Infrastruktur gefährden könnten - eine große Bedeutung zu (Hassel et al. 2014; Eleschová und Beláň 2008; Ri- 
naldi et al. 2002). Eine Maßnahme, um die Versorgung abzusichern, ist das Vorhandensein unabhängiger redundanter Systeme, die die Vulnerabilität gegen äußere Einflüsse, wie etwa extreme Wetterbedingungen, erhöhen. Die zugehörigen Maßnahmen sollten dabei stets individuell an die vorherrschenden Rahmenbedingungen angepasst werden (DVGW 2018). Bei der Planung muss jedoch immer bedacht werden, dass viele Störungen nicht monokausal auf eine Ursache zurückgeführt werden können. In vielen Fällen ist das Zusammenwirken unterschiedlicher Faktoren dafür verantwortlich, wobei dann von kumulativen Extremereignissen gesprochen wird (Hildebrandt et al. 2017). So können nasse Böden in Verbindung mit einem Sturmereignis die Standfestigkeit belaubter Bäume herabsetzen und dadurch das Schadenspotenzial für Teile der Versorgungsinfrastruktur durch umstürzende Bäume erhöhen.

Klimatische Veränderungen können allerdings auf vielfältige Art und Weise einzelne Elemente der kritischen Infrastruktur - angefangen von einem Stromverteilerkasten bis hin zur Steuerung der Kläranlage oder dem Anfahrtsweg zu einem Wasserwerk - negativ beeinflussen. Starkregenereignisse oder extrem trockene und überdurchschnittlich warme Jahre wie 2018 lassen schon heute die Anfälligkeiten in vielen Teilbereichen der kritischen Infrastruktur erkennen. Die Intensivierung extremer Trockenheit kann den Boden so beeinträchtigen, dass an unterirdisch verlegten Stromkabeln Risse entstehen (sog. „Sommerfrost“) (Böhmer et al. 2020) oder die Brandgefahr in Wäldern auf Wasserwerksgeländen steigt (DVGW 2018). Überflutungen und Sturmschäden können an Gebäuden und Bauwerken Schäden verursachen, was zu kontrollierten oder unkontrollierten Abschaltungen führen kann. Davon können sämtliche elektronisch gesteuerten Anlagen und mitunter die gesamte Kommunikation betroffen sein. Durch die weiterhin steigenden Temperaturen und die Zunahme der Anzahl und Intensität von extremen Wetterereignissen, wird sich die Situation sehr wahrscheinlich weiter verschärfen.

\section{Den Herausforderungen begegnen}

Die Folgen des Klimawandels werden in Verbindung mit dem demographischen Wandel, der fortschreitenden Urbanisierung, einem sich ändernden Wasserverbrauch und einem zunehmenden temporären Wasserstress Wasserversorger vor neue Herausforderungen stellen. Um diesen vielfältigen und sektorübergreifenden Anforderungen begegnen zu können, werden integrative Ansätze benötigt, die da$\mathrm{zu}$ beitragen die isolierten sektoralen Betrachtungsweisen zu überwinden. Nur auf dieser Grundlage wird es möglich sein, sowohl den direkten Folgen durch Extremwetterereignisse entgegenwirken zu können, als auch das Überspringen von Teilsystemausfällen zwischen unterschiedlichen Sek- toren (sogenannter Dominoeffekt) oder den zunehmenden Ausfall immer neuer Teilsysteme und die damit verbundenen immer stärker werdenden Auswirkungen (sogenannter Kaskadeneffekt) abmildern oder vermeiden zu können.

Ein wichtiger Baustein ist hierbei der Aufbau redundanter, klimaresilienter Systemkomponenten, um Engpässe oder Teilsystemausfälle kompensieren zu können.

Ansätze zur Identifikation möglicher Schwachstellen im Gesamtsystem bieten partizipatorische Modellierungsansätze mit einem Fokus auf den spezifischen Bedarfen aus der Perspektive aller relevanten Praxisakteure. Aufbauend auf dieser mehrdimensionalen, systemischen Sichtweise kann im Anschluss eine impulsgebende Sensibilisierung von beispielsweise den Betreibern kritischer Infrastrukturelemente erfolgen, um ganzheitliche Maßnahmen zur Anpassung an den Klimawandel umsetzen zu können (Groth und Seipold 2020). Neben der Anpassung der Anlagen an die unterschiedlichen möglichen Folgen des Klimawandels ist die Sicherstellung einer auch bei Extremwetterereignissen funktionsfähigen Ver- und Entsorgungsinfrastruktur von zentraler Bedeutung (Groth et al. 2018; Groth und Rose 2017).

Für Deutschland ist generell von einer klimawandelbedingten Betroffenheit der kritischen Infrastruktur auszugehen, der man grundsätzlich vor allem durch technische Anpassungsmaßnahmen entgegenwirken kann. Allerdings müssen dazu rechtzeitig zielgerichtete Maßnahmen ergriffen werden. Deshalb sollte schon jetzt sowohl auf der Ebene einzelwirtschaftlicher Optimierungen von Unternehmensstandorten als auch bei anstehenden überregionalen Infrastrukturmaßnahmen die Ergebnisse regionaler Klimaprojektionen Berücksichtigung finden. Damit könnte die Transformation $\mathrm{zu}$ klimaresilienten und $\mathrm{CO}_{2}$-neutralen Standorten auch zur Beseitigung entsprechender Schwachstellen und letztlich zur langfristig klimawandelangepassten Auslegung der Infrastruktur genutzt werden. Ein zentraler Schlüssel liegt hierbei in der Ausgestaltung regulatorischer Rahmenbedingungen. Hier gilt es nicht nur, bestehende Vorgaben auf den Prüfstand zu stellen und weiterzuentwickeln, sondern auch neue Wege zu gehen (Groth et al. 2018).

Auch wenn die Betroffenheiten kritischer Infrastrukturelemente in einzelnen Sektoren in der Theorie bekannt sind (DVGW 2018; Groth et al. 2018), bestehen noch große Wissenslücken und umfassender Forschungsbedarf zu den Auswirkungen von Multi-Gefahren auf stetig komplexer werdende Systeme, einschließlich möglicher Domino- und Kaskadeneffekte sowie der Entwicklung passender Indikatoren basierend auf der Kombination von Klimaparametern und weiterer Umweltinformationen.

Dies gilt vor allem für Projektionen der Grundwasserneubildung und sich daraus ergebender Veränderungen der Grundwasserganglinien und -ressourcen sowie die notwendige Berücksichtigung von Klimawandelfolgen im Zuge der zukünftigen Ausgestaltung regulatorischer Rahmenbedin- 
gungen und den damit verbundenen ökonomischen Anreizsetzungen zur Transformation der kritischen Infrastruktur, beispielsweise durch ein Klassifizierungssystem für ökonomische Aktivitäten (,Taxonomie“) auch für die Finanzierung von Infrastrukturen oder die Einführung spezifischer Berichtspflichten für Betreiber kritischer Infrastrukturen zum Umgang mit Risiken und Chancen eines sich ändernden Klimas.

Eine Möglichkeit hierfür wäre die Einführung spezifischer Berichtspflichten für Betreiber kritischer Energieinfrastrukturen zur Einschätzung von - und insbesondere auch dem Umgang mit - heutigen und zukünftigen Risiken und Chancen eines sich ändernden Klimas. Dies könnte sich beispielweise an der Berichtspflicht aufgetretener Versorgungsunterbrechungen orientieren, die die Energienetzbetreiber an die Bundesnetzagentur berichten. Darüber hinaus sind erweiterte Systembetrachtungen notwendig, die über isolierte Einzelbetrachtungen hinausgehen und mögliche Wechselwirkungen zwischen grundlegend unterschiedlichen Infrastrukturbereichen beinhalten, einschließlich der Betrachtungen zu klimawandelbedingten gegenseitigen Beeinflussungen und den sich daraus ergebenden Kaskadenund Dominoeffekten.

Funding Open Access funding enabled and organized by Projekt DEAL.

Open Access Dieser Artikel wird unter der Creative Commons Namensnennung 4.0 International Lizenz veröffentlicht, welche die Nutzung, Vervielfältigung, Bearbeitung, Verbreitung und Wiedergabe in jeglichem Medium und Format erlaubt, sofern Sie den/die ursprünglichen Autor(en) und die Quelle ordnungsgemäß nennen, einen Link zur Creative Commons Lizenz beifügen und angeben, ob Änderungen vorgenommen wurden.

Die in diesem Artikel enthaltenen Bilder und sonstiges Drittmaterial unterliegen ebenfalls der genannten Creative Commons Lizenz, sofern sich aus der Abbildungslegende nichts anderes ergibt. Sofern das betreffende Material nicht unter der genannten Creative Commons Lizenz steht und die betreffende Handlung nicht nach gesetzlichen Vorschriften erlaubt ist, ist für die oben aufgeführten Weiterverwendungen des Materials die Einwilligung des jeweiligen Rechteinhabers einzuholen.

Weitere Details zur Lizenz entnehmen Sie bitte der Lizenzinformation auf http://creativecommons.org/licenses/by/4.0/deed.de.

\section{Literatur}

BayLfW - Bayerisches Landesamt für Wasserwirtschaft: Informationsbericht des Bayerischen Landesamtes für Wasserwirtschaft, Heft 2/05 München, 162 S (2005). https://www.lfu.bayern. de/wasser/gewaesserkundliche_berichte/sonderberichte/doc/sb_ niedrigwasserperoide2003.pdf, Zugegriffen: 1. Sept. 2020

BBK - Bundesamt für Bevölkerungsschutz und Katastrophenhilfe: 10 Jahre „KRITIS-Strategie“. Einblicke in die Umsetzung der Nationalen Strategie zum Schutz Kritischer Infrastrukturen, S. 110 (2020)

Bender, S., Jacob, D.: Die Aussagekraft von Klimaprojektionen für zukünftige Herausforderungen der Trinkwasserversorgung in Deutschland. GWF Wasser Abwasser 04, 362-368 (2016)
Bender, S., Butts, M., Hagemann, S., Smith, M., Vereecken, H., Wendland, F.: Der Einfluss des Klimawandels auf die terrestrischen Wassersysteme in Deutschland. Eine Analyse ausgesuchter Studien der Jahre 2009 bis 2013. Climate Service Center GermanyReport, Bd. 29., S. 77 (2017)

Bender, S., Groth, M., Otto, J., Pfeifer, S., Seipold, P.: Regionale Klimainformationen für Rostock. GERICS City Series, Bd. 03., S. 25 (2019)

Bender, S., Groth, M., Pfeifer, S., Raub, T.: Regionale Klimainformationen für Bremen - Überprüfung, Erweiterung, Anwendung. GERICS City Series, Bd. 02., S. 28. (2018)

van den Besselaar, E.J.M., Klein Tank, A.M.G., Buishand, T.A.: Trends in European precipitation extremes over 1951-2010. Int. J. Climatol. 33, 2682-2689 (2012)

Birkmann, J., Bach, C., Guhl, S., Witting, M., Welle, T., Schmude, M.: State of the Art der Forschung zur Verwundbarkeit Kritischer Infrastrukturen am Beispiel Strom/Stromausfall. Schriftenreihe Sicherheit, Bd. 2., S. 135 (2010)

Böhmer, H., Brinkmann-Wicke, T., Sell, S., Simon, J., Tebben, C.: VHV-Bauschadenbericht Bd. 2019/20. Fraunhofer IRB, Hannover, S. 240 (2020)

Brahmer, G.: Klimawandel und seine Konsequenzen für die Wasserwirtschaft in Hessen. In: Fachvorträge KLIWA-Symposium am 25. und 26.10.2006 in Stuttgart KLIWA-Heft, Bd. 10, S. 211-222. (2006in)

Brasseur, G.P., Jacob, D., Schuck-Zöller, S. (Hrsg.): Klimawandel in Deutschland - Entwicklung, Folgen, Risiken und Perspektiven. Springer, Berlin, Heidelberg, New York, S. 368 (2016)

Collins, M., Knutti, R., Arblaster, J., Dufresne, J.-L., Fichefet, T., Friedlingstein, P., Gao, X., Gutowski, W.J., Johns, T., Krinner, G., Shongwe, M., Tebaldi, C., Weaver, A.J., Wehner, M.: Longterm climate change: projections, commitments and irreversibility. In: Stocker, T.F., Qin, D., Plattner, G.-K., Tignor, M., Allen, S.K., Boschung, J., Nauels, A., Xia, Y., Bex, V., Midgley, P.M. (Hrsg.) Climate Change 2013: The Physical Science Basis. Contribution of Working Group I to the Fifth Assessment Report of the Intergovernmental Panel on Climate Change, S. 1029-1136. Cambridge University Press, Cambridge, New York (2013)

Cremades, R., Mitter, H., Tudose, N.C., Sanchez-plaza, A., Graves, A., Broekman, A., Bender, S., Giupponi, C., Koundouri, P., Bahri, M., Cheval, S., Cortekar, J., Moreno, Y., Melo, O., Karner, K., Ungurean, C., Davidescu, S.O., Kropf, B., Brouwer, F., Mirabelin, M.: Ten principles to integrate the water-energy-land nexus with climate services for co-producing local and regional integrated assessments. Sci. Total Environ. 693, 133662 (2019). https:// doi.org/10.1016/j.scitotenv.2019.133662

Deutschländer, T., Mächel, H.: Temperatur inklusive Hitzewellen. In: Brasseur, G.P., Jacob, D., Schuck-Zöller, S. (Hrsg.) Klimawandel in Deutschland - Entwicklung, Folgen, Risiken und Perspektiven, S. 47-56. Springer, Berlin, Heidelberg, New York (2016)

Döll, P.: Vulnerability to the impact of climate change on renewable groundwater resources: a global-scale assessment. Special Issue: Focus on groundwater resources, climate and vulnerability. Environ. Res. Lett. 4, 1-12 (2009)

DVGW: Betriebliche Aspekte im Zusammenhang mit Extremwetterereignissen bei Wassergewinnungsanlagen. DVGW-Information Wasser, Bd. 96. DVGW, Bonn, S. 34 (2018)

DWD - Deutscher Wetterdienst: Nationaler Klimareport Bd. 3. DWD, Offenbach a.M., S. 46 (2017)

Eleschová, Z., Belán̆, A.: Blackout in the power system. ATP J. Plus2 2008, 58-60 (2008))

Euler, C., Gerdes, H., Kämpf, M.: Sustainable groundwater management and climate change. Water Waste 3, 10-12 (2009)

Eusgeld, I., Nan, C., Dietz, S.: System-of-systems approach for interdependent critical infrastructures. Reliab. Eng. Syst. Saf. 96(6), 679-686 (2011) 
Ferguson, G., Gleeson, T.: Vulnerability of coastal aquifers to groundwater use and climate change. Nature Clim Change 2, 342-345 (2012)

Feseker, T.: Numerical studies on saltwater intrusion in a coastal aquifer in northwestern Germany. Hydrogeol J 15, 267-279 (2007)

Forzieri, G., Bianchi, A., eSilva, F.B., Marin Herrera, M.A., Leblois, A., Lavalle, C., Aerts, J.C.J.H., Feyen, L.: Escalating impacts of climate extremes on critical infrastructures in Europe. Glob Environ Change 48, 97-107 (2018)

Graumann, S.: Energie- und Wasserversorger noch verhalten bei der Digitalisierung. Energ Wasser Praxis Kompakt 4, 6-9 (2017)

Green, T.R., Taniguchi, M., Kooi, H., Gurdak, J.J., Allen, D.M., Hiscook, K.M., Treidel, H., Aureli, A.: Beneath the surface of global change: impacts of climate change on groundwater. J. Hydrol. Reg. Stud. 405(3-), 532-560 (2011)

Groth, M.: Klimapolitik - Stand des Wissens, Zielverfehlungen und Handlungsnotwendigkeiten. Wirtschaftsdienst 99(12), 819-824 (2019)

Groth, M., Rose, J.: Infrastrukturen (Energie- und Wasserversorgung). In: Meinke, I., von Storch, H., Claußen, M. (Hrsg.) 2. Hamburger Klimabericht - Wissen über Klima, Klimawandel und Auswirkungen in Hamburg und Norddeutschland, S. 193-208. (2017)

Groth, M., Seipold, P.: Business strategies and climate change-Prototype development and testing of a user specific climate service product for companies. In: Leal Filho, W., Jacob, D. (Hrsg.) Handbook of Climate Services, S. 51-66. Spinger, Berlin, Heidelberg, New York (2020)

Groth, M., Bender, S., Cortekar, J., Remke, T., Stankoweit, M.: Auswirkungen des Klimawandels auf den Energiesektor in Deutschland. Z. Umweltpolit. Umweltr. 3, 324-355 (2018)

Groth, M., Cortekar, J., Bender, S.: The relevance of cascading effects for adapting critical infrastructures to climate change. In: Proc. Adaptation Futures 2016 Science Abstracts, Bd. 10. (2016)

Hassel, H., Johansson, J., Cedergren, A., Svegrup, L., Arvidsson, B.: Method to study cascading effects. Deliverable 2.1, Modelling of dependencies and cascading effects for emergency management in crisis situations (CascEff Project), 40 S. (2014). http:// casceff.eu/media2/2016/02/D2.1-Deliverable_Final_Ver2_PU. pdf, Zugegriffen: 10. Sept. 2020

Hattermann, F.F., Post, J., Krysanova, V., Conradt, T., Wechsung, F.: Assessment of water availability in a Central European river basin (Elbe) under climate change. Adv Clim Change Res 4, 42-50 (2008)

Hergesell, M.: Auswirkungen der Trockenperiode 2016-2017 auf das Grundwasser. In: Hessisches Landesamt für Naturschutz, Umwelt und Geologie - Jahresbericht 2017, S. 63-71. (2017)

Hildebrandt, S., Schuler, J., Krämer, C., Steinhäußer, R.: Berücksichtigung kumulativer Wirkungen in der Umweltplanung. Nat. Landsch. 92(5), 209-213 (2017)

HLUG - Hessisches Landesamt für Umwelt und Geologie: Klimawandel und Wasser. Reihe Klimawandel in Hessen, 23 S. (2014). https://www.hlnug.de/fileadmin/dokumente/klima/klimawandel_ wasser.pdf, Zugegriffen: 3. Sept. 2020

IPCC: Summary for Policymakers. In: Edenhofer, O., Pichs-Madruga, R., Sokona, Y., Farahani, E., Kadner, S., Seyboth, K., Adler, A., Baum, I., Brunner, S., Eickemeier, P., Kriemann, B., Savolainen, J., Schlömer, S., von Stechow, C., Zwickel, T., Minx, J.C. (Hrsg.) Climate Change 2014: Mitigation of Climate Change. Contribution of Working Group III to the Fifth Assessment Report of the Intergovernmental Panel on Climate Change, S. 30. Cambridge University Press, Cambridge, New York (2014)

IPCC: Global warming of $1.5{ }^{\circ} \mathrm{C}$. Special Report. IPCC, Geneva, S. 616 (2018). IPCC with World Meteorological Organisation (WMO) and United Nations Environmental Program (UNEP)

Jacob, D., Bülow, K., Kotova, L., Moseley, C., Petersen, J., Rechid, D.: Regionale Klimaprojektionen für Europa und Deutschland: Ensemble Simulationen für die Klimafolgenforschung. CSC Report,
Bd. 6. Climate Service Center Germany, Selbstverlag, Hamburg, S. 48 (2012)

Jacob, D., Petersen, J., Eggert, B., Alias, A., Christensen, A.B., Bouwer, L.M., Braun, A., Colette, A., Dequue, M., Georgievski, G., Georgopoulou, E., Gobiet, A., Menut, L., Nikulin, G., Hänsler, A., Hempelmann, N., Jones, C., Keuler, K., Kovats, S., Kroner, N., Kotlarski, S., Kriegsmann, A., Martin, E., van Meijgaard, E., Moeseley, C., Pfeifer, S., Preuschmann, S., Radermacher, C., Radtke, K., Rechid, D., Rounsevell, M., Samuelsson, P., Somot, S., Soussanna, J.-F., Teichmann, C., Valentini, R., Vautard, R., Weber, B., Yiou, P.: EURO-CORDEX: new high-resolution climate change projections for European impact research. Reg. Environ. Chang. 14(2), 563-578 (2014)

Kunstmann, H., Fröhle, P., Hattermann, F.F., Marx, A., Smiatek, G., Wanger, C.: Wasserhaushalt. In: Brasseur, G.P., Jacob, D., Schuck-Zöller, S. (Hrsg.) Klimawandel in Deutschland - Entwicklung, Folgen, Risiken und Perspektiven, S. 161-172. Springer, Berlin, Heidelberg, New York (2016)

Kunz, M., Mohr, S., Werner, P.: Niederschlag. In: Brasseur, G.P., Jacob, D., Schuck-Zöller, S. (Hrsg.) Klimawandel in Deutschland - Entwicklung, Folgen, Risiken und Perspektiven, S. 57-66. Springer, Berlin, Heidelberg, New York (2016)

Laugé, A., Hernantes, J., Sarriegi, J.M.: Critical infrastructure dependencies: A holistic, dynamic and quantitative approach. Int. J. Crit. Infrastruct. Prot. 8, 16-23 (2015)

Malitz, G., Beck, C., Grieser, J.: Veränderung der Starkniederschläge in Deutschland (Tageswerte der Niederschlagshöhe im 20. Jahrhundert). In: Lozán, J.L., Graß1, H., Hupfer, P., Karbe, L., Schönwiese, C.D. (Hrsg.) Warnsignal Klima: Genug Wasser für alle?, 3. Aufl., S. 311-316. (2011)

Meinshausen, M., Smith, S.J., Calvin, K., Daniel, J.S., Kainuma, M.L.T., Lamarque, J.-F., Matsumoto, K., Montzka, S., Raper, S., Riahi, K., Thomson, A., Velders, G.J.M., van Vuuren, D.P.: The RCP greenhouse gas concentrations and their extensions from 1765-2300. Clim Change 109, 213-241 (2011)

Merkel, W., Staben, N.: Sichere Wasserversorgung im Klimawandel Dnyaklim-Broschüre, S. 19 (2014)

Mikellidou, C.V., Shakou, L.M., Boustras, G., Dimopoulos, C.: Energy critical infrastructures at risk from climate change: a state of the art review. Saf Sci 110(Part C), 110-120 (2018)

Mohr, S., Kunz, M.: Recent trends and variabilities of convective parameters relevant for hail events in Germany and Europe. Atmos. Res. 123, 211-228 (2013)

Moss, R.H., Edmonds, J.A., Hibbard, K.A., Manning, M.R., Rose, S.K., van Vuuren, D.P., Carter, T.R., Emori, S., Kainuma, M., Kram, T., Meehl, G.A., Mitchell, J.F., Nakicenovic, N., Riahi, K., Smith, S.J., Stouffer, R.J., Thomson, A.M., Weyant, J.P., Wilbanks, T.J.: The next generation of scenarios for climate change research and assessment. Nature 463, 747-756 (2010)

Olfert, A., Brunnow, B., Schiller, G., Walther, J., Hirschnitz-Garbers, M., Langsdorf, S., Hinzmann, M., Hölscher, K., Wittmayer, J.: Nachhaltigkeitspotenziale innovativer, gekoppelter Infrastrukturen - Teilbericht des Vorhabens: Transformation hin zu nachhaltigen, gekoppelten Infrastrukturen. UBA Texte, Bd. 99/2020., S. 305 (2020)

Osmancevic, E., Engelfried, M., Friedmann, R.: Erhöhte Temperaturen in Trinkwasser-Versorgungssystemen. Energ. Wasser Prax. 09/2018, 58-63 (2018)

Pfeifer, S., Bülow, K., Gobiet, A., Hänsler, A., Mudelsee, M., Otto, J., Rechid, D., Teichmann, C., Jacob, D.: Robustness of ensemble climate projections analyzed with climate signal maps: seasonal and extreme precipitation for Germany. Atmosphere 6, 677-698 (2015)

ReKliEs-De: ReKliEs-De Ergebnisbericht, 76 S. (2017). https://doi. org/10.2312/WDCC/ReKliEsDe_Ergebnisbericht. Zugegriffen: 07.05 .2020 
Riedel, T.: Temperature-asociated changes in groundwater quality. J. Hydrol. 572, 206-212 (2019)

Rinaldi, S.M., Peerenboom, J.P., Kelly, T.K.: Identifying, understanding, and analyzing critical infrastructure interdependencies. IEEE Control. Syst. 21(6), 11-25 (2002)

Stankoweit, M., Groth, M., Jacob, D.: On the heterogeneity of the economic value of electricity distribution networks: an application to Germany. In: Working Paper Series in Economics, University of Lueneburg Working Paper, Bd. 371. (2017)

Tart, S., Groth, M., Seipold, P.: Market demand for climate services: an assessment of users' needs. Clim. Serv. 17, 100-109 (2020)

Trömel, S., Schönwiese, C.-D.: Probability change of extreme precipitation observed from 1901-2000 in Germany. Theor. Appl. Climatol. 87, 29-39 (2007)

Umweltbundesamt: Monitoringbericht 2019 zur Deutschen Anpassungsstrategie an den Klimawandel. Bericht der Interministeriellen Arbeitsgruppe Anpassungsstrategie der Bundesregierung. Umweltbundesamt, Dessau-Roßlau, S. 272 (2019)

Wagner, S., Berg, P., Schädler, G., Kunstmann, H.: High resolution regional climate model simulations for Germany: Part II-projected climate changes. Clim Dyn 40, 415-427 (2013)
Whitehead, P.G., Wilby, R.L., Battarbee, R.W., Kernan, M., Wade, A.J.: A review of the potential impacts of climate change on surface water quality. Hydrol. Sci. J. 54(1), 101-123 (2009)

World Economic Forum: The Global Risks Report 2020, 15. Aufl. World Economic Forum, Geneva, S. 102 (2020)

Zebisch, M., Grothmann, T., Schröter, D., Hasse, C., Fritsch, U., Cramer, W.: Klimawandel in Deutschland. Vulnerabilität und Anpassungsstrategien klimasensitiver Systeme. Climate Change, Bd. 08/05., S. 203 (2005)

Zolina, O., Simmer, C., Kapala, A., Bachner, S., Gulev, S., Maechel, H.: Seasonally dependent changes of extremes over Germany since 1950 from a very dense observational network. J. Geophys. Res. 113, 1-17 (2008)

Hinweis des Verlags Der Verlag bleibt in Hinblick auf geografische Zuordnungen und Gebietsbezeichnungen in veröffentlichten Karten und Institutsadressen neutral. 